\title{
Spatial origin of Galactic cosmic rays in diffusion models
}

\section{Exotic primary cosmic rays}

\author{
D. Maurin ${ }^{1,2}$ and R. Taillet ${ }^{1,3}$ \\ 1 Laboratoire de Physique Théorique LAPTH, 74941 Annecy-le-Vieux, France \\ 2 Institut d'Astrophysique de Paris, 98 bis Bd Arago, 75014 Paris, France \\ 3 Université de Savoie, 73011 Chambéry, France
}

Received 6 December 2002 / Accepted 27 March 2003

\begin{abstract}
In a companion paper, we investigated the question of the spatial origin of the cosmic rays detected in the Solar neighborhood, in the case of standard sources located in the Galactic disk. There are some reasons to believe that there may also be a large number of sources located in the halo, for example if the Galactic dark matter is made of supersymmetric particles or if Primordial Black Holes are present. These exotic sources could enhance the $\bar{p}, \bar{d}$ or positrons above the standard background, indicating the existence of new physics. The spatial distribution of these hypothetical sources, though an important ingredient to evaluate these exotic signals, is poorly known. The aim of this paper is to point out that this discussion should not be disconnected from that of the propagation properties in the Galaxy. More precisely, we determine the regions of the halo from which a significant fraction $f$ of cosmic rays antiprotons and antideuterons detected in the Solar neighborhood were emitted (we refer to these regions as $f$-volumes), for different sets of propagation parameters consistent with $\mathrm{B} / \mathrm{C}$ data, as derived in Maurin et al. (2002). It is found that some of them lead to rather small $f$-volumes, indicating that the exotic cosmic rays could have a local origin (in particular for a small diffusive halo or a large Galactic convective wind), coming from the Solar neighborhood or the Galactic center region. It is also found that the dark matter density enhancement (spike) due to the accretion around the central supermassive black hole gives a negligible contribution to the exotic charged particle signal on Earth. The case of electrons and positrons is also discussed.
\end{abstract}

Key words. ISM: cosmic rays - cosmology: dark matter - black hole physics

\section{Introduction}

A great amount of work has been done these last twenty years on the astrophysical signatures that could unravel new physics. In the eighties, there were great hopes that the antiproton signal, which showed an excess at an energy of a few hundreds of $\mathrm{MeV}$ in the first balloon experiments, could be such a signature. However, this hope was swept away by the progress in measurements - see e.g. BESS (Orito et al. 2000; Maeno et al. 2001) or HEAT (Beach et al. 2001) and CAPRICE (Boezio et al. 2001) at higher energy - and a better determination of the cosmic ray propagation parameters (see e.g. Maurin et al. 2002). It was shown that the measured antiproton flux was indeed compatible with the sole secondary standard spallative production (Bergström et al. 1999b; Donato et al. 2001) (see the first paper for a comprehensive historical discussion and a panel of references dealing with exotic antiproton production).

Donato et al. (2000) showed that the antideuteron $(\bar{d})$ signal could lead to a clearer signature of SUSY. However, as discussed in many other studies on SUSY antiprotons

Send offprint requests to: $\mathrm{R}$. Taillet, e-mail: taillet@lapp.in2p3.fr
(Rudaz \& Stecker 1988; Stecker \& Tylka 1989; Jungman \& Kamionkowski 1994; Bottino et al. 1995, 1998; Wells et al. 1999; Bergström et al. 1999b), the indeterminacy in the dark matter distribution, as well as its possible clumpiness (Bergström et al. 1999a), might severely change the conclusions. In contrast, the Hawking evaporation of Primordial Black Holes (PBH) could also yield a new source of cosmic rays (Maki et al. 1996), but the precise shape of the dark matter in this case is not crucial (Barrau et al. 2002, 2003). Nevertheless, in the latter case, it was shown that even considering only the propagation parameters giving a good fit to $\mathrm{B} / \mathrm{C}$ data, the remaining degeneracy for example in the diffusive halo height has sizeable effects on the primary flux (Barrau et al. 2002).

Hence, at least two different phenomena can affect the conclusions reached in papers dealing with exotic flux calculations. The first one, related to the spatial distribution of SUSY sources, is usually thoroughly discussed (Bergström et al. 1999b), but the second point - namely the influence of various propagation parameters - is generally skipped, due to the simplicity of the propagation models used. The aim of the paper is not to compare the predicted $\bar{p}, \bar{D}$ fluxes with 
observations for different series of models, but rather to point out which characteristics of the models actually play a role, in order to give some physical insights and milestones for studies specifically devoted to exotic flux evaluations.

We apply the method described in Taillet \& Maurin (2003) to determine the volumes from which a fraction $f$ of cosmic rays reaching the Solar neighborhood were emitted, or equivalently the volumes that contribute to the fraction $f$ of the total flux detected in the Solar neighborhood. These volumes will be referred to as the $f$-volumes throughout the paper.

We find that depending on the diffusion parameters (evaluated from a systematic study of standard CR, Maurin et al. 2002) as well as on the source spatial distribution, the spatial origin of cosmic rays may be quite local, the particles detected in the Solar neighborhood having mostly been created a few kpc away from the Solar neighborhood in some cases, or a few kpc away from the Galactic center in others.

\section{Evaluation of the $f$-volumes}

In a companion paper (Taillet \& Maurin 2003), we presented a method to compute the region from which a cosmic ray detected in the Solar neighborhood has a given probability of originating. This method was applied to standard sources located in the disk, and we now use it for (exotic) sources in the halo. A schematic view of our model is presented in Fig. 1 where the isothermal dark matter profile has been superimposed on the Galaxy to compare their typical scales (the reader is referred to Taillet \& Maurin 2003 for all the details concerning the model, such as the functional form of the galactic wind and the geometry of the box). The probability that a particle detected in the Solar neighborhood was emitted from any finite volume $\mathcal{V}$ can be computed as

$\mathcal{P}\left\{\mathcal{V} \mid \boldsymbol{r}_{\mathrm{o}}\right\}=\frac{\int_{\mathcal{V}} w\left(\boldsymbol{r}_{\mathrm{s}}\right) N_{\mathrm{r}_{\mathrm{s}}}\left(\boldsymbol{r}_{\mathrm{o}}\right) \mathrm{d}^{3} \boldsymbol{r}_{\mathrm{s}}}{\int_{\mathcal{V}_{\mathrm{tot}}} w\left(\boldsymbol{r}_{\mathrm{s}}\right) N_{\mathrm{r}_{\mathrm{s}}}\left(\boldsymbol{r}_{\mathrm{o}}\right) \mathrm{d}^{3} \boldsymbol{r}_{\mathrm{s}}}$,

where the source distribution $w\left(\boldsymbol{r}_{\mathrm{s}}\right)$ has been introduced and $N_{\mathrm{r}_{\mathrm{s}}}\left(\boldsymbol{r}_{\mathrm{o}}\right)$ is the density in $\boldsymbol{r}_{\mathrm{o}}$ resulting from a point source located in $\boldsymbol{r}_{\mathrm{s}}$. In this paper, we are interested in determining $f$-volumes, i.e. volumes $\mathcal{V}(f)$ from which a given fraction $f$ of cosmic rays detected in the Solar neighborhood were emitted. They are defined by

$\mathcal{P}\left\{\mathcal{V}(f) \mid \boldsymbol{r}_{\mathrm{o}}\right\}=f$.

Actually, even for a given value of $f$, there are many different volumes, delimited by different closed surfaces, fulfilling this condition. We focus on the smallest of these volumes, precisely delimited by an isodensity surface. Monte Carlo integration is then particularly well adapted to evaluate the integrals in Eq. (1). In a typical run, $\sim 10^{6}$ points are required to reach a $\$ 0.5 \%$ convergence and the integral is performed inside all isodensity surfaces at once, so that the $f$-volume defined by Eq. (2) are simple to recover.

\subsection{Influence of the propagation parameters}

The quantity $N_{\mathrm{r}_{\mathrm{s}}}\left(\boldsymbol{r}_{\mathrm{o}}\right)$ appearing in Eq. (1) is evaluated by solving the diffusion equation with a point-like source, in the geometry depicted in Fig. 1. Propagation is affected, at different levels, by three effects: escape, galactic wind and spallations. First, escape happens when a particle reaches one of the boundaries of the diffusive volume. As discussed in the companion paper, this limits the range from which cosmic rays can travel to the Solar neighborhood. It was also shown that the side boundary plays only a minor role, and one can assume that the box has an infinite radial extension. Second, a convective wind $V_{\mathrm{c}}$ directed out from the Galactic plane blows the charged nuclei away, so that it is more difficult to reach the plane from high $z$ sources. Finally, spallations may happen when a nucleus crossing the thin disk interacts with the interstellar matter. The nuclei are then destructed at a rate $\Gamma_{\text {inel }}=2 h n_{\mathrm{ISM}} \cdot v \cdot \sigma_{\text {inel }}$. A particle emitted from a remote source is more affected by spallations as it is likely to have crossed the disk many times before reaching the Solar neighborhood. In the companion paper, this effect was shown to be important for heavy species created in the disk. Here, we focus on very light species, having smaller cross-sections, which are mostly created in the halo. They are affected by the wind in the whole halo, i.e. from the moment of their creation, whereas they are only affected by spallations when they cross the disk, which is less likely for halo sources than for disk sources. As a result, spallations play only a minor role in the present study (this effect is nevertheless included in our treatment).

When these three effects are taken into account, the density in $O$ due to a Dirac source $\delta\left(\boldsymbol{r}-\boldsymbol{r}_{\mathrm{s}}\right)$ can be computed. Because of the cylindrical symmetry present for an infinite disk, it is equivalent to consider a source term $\delta\left(z-z_{\mathrm{s}}\right) \delta\left(r-r_{\mathrm{s}}\right) / 2 \pi r_{\mathrm{s}}$, which leads to

$N_{\left(r_{\mathrm{s}}, z_{\mathrm{s}}\right)}(0)=\mathrm{e}^{-z_{\mathrm{s}} / r_{\mathrm{w}}} \sum_{i=1}^{\infty} \frac{J_{0}\left(\zeta_{i} r_{\mathrm{s}} / R\right)}{\pi J_{1}^{2}\left(\zeta_{i}\right) R^{2} A_{i}} \times \frac{\sinh \left[S_{i}\left(L-z_{\mathrm{s}}\right) / 2\right]}{\sinh \left(S_{i} L / 2\right)}$

with

$S_{i}=\sqrt{\frac{4}{r_{\mathrm{w}}}+\frac{4 \zeta_{i}^{2}}{R^{2}}}$ and $A_{i}=K\left(\frac{2}{r_{\mathrm{sp}}}+\frac{2}{r_{\mathrm{w}}}+S_{i} \operatorname{coth}\left[\frac{S_{i} L}{2}\right]\right)$

and where the parameters

$\left\{\begin{array}{l}r_{\mathrm{w}} \equiv \frac{2 K}{V_{\mathrm{c}}} \approx 5.87 \mathrm{kpc} \times \frac{K(E)}{0.03 \mathrm{kpc}^{2} \mathrm{Myr}^{-1}} \frac{10 \mathrm{~km} \mathrm{~s}^{-1}}{V_{\mathrm{c}}}, \\ r_{\mathrm{sp}} \equiv \frac{2 K}{2 h \Gamma_{\text {inel }}} \approx \frac{3.17 \mathrm{kpc}}{\beta} \times \frac{K(E)}{0.03 \mathrm{kpc}^{2} \mathrm{Myr}^{-1}} \frac{100 \mathrm{mb}}{\sigma},\end{array}\right.$

give the order of magnitude of the typical distance over which the associated process affects propagation. In practice, large values of $R$ have been used in Eq. (3) so that the hypothesis $R \rightarrow \infty$ is actually recovered. The effect of escape, wind and spallations are compared in Fig. 2, which shows the shape of the isodensity surfaces for two values $L=5 \mathrm{kpc}$ (left panels) and $L=10 \mathrm{kpc}$ (right panels), and for typical values of $r_{\mathrm{w}}$ and $r_{\mathrm{sp}}$. The value $r_{\mathrm{sp}}=3.17 \mathrm{kpc}$ has been retained because it corresponds to the antideuteron destruction cross section for a typical value of the diffusion coefficient $K=0.03 \mathrm{kpc}^{2} \mathrm{Myr}^{-1}$. In the upper panels, one can see the shrinking of the contours in the vicinity of the disk, due to the effect of spallations. The effect of the wind is rather to flatten the contours, 


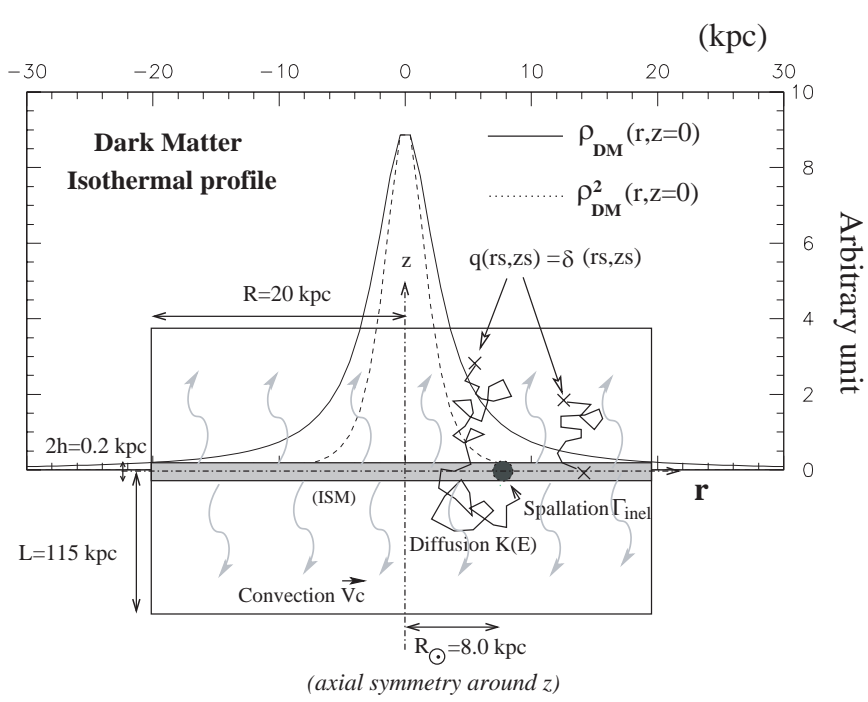

Fig. 1. Schematic view of our Galaxy: diffusive and convective propagation plus spallations in the thin disk. Effective primary exotic sources follow either the dark matter profile or its square (isothermal profile is depicted).

as can be seen in the lower panels. The probability density also decreases more rapidly when convection or spallations are included than when diffusion alone is considered. As a result, the $99 \%$-volumes are reduced, as indicated by the thick lines. It is thus of importance to use realistic values for $K(E), L$ and $V_{\mathrm{c}}$ in order to give confident $f$-volumes for real situations $\left(\sigma_{\text {inel }}\right.$ is not a free parameter, it solely depends on the species we consider).

To summarize the previous results about the origin of exotic primaries in diffusion/convection/spallation models: i) the pure diffusive regime provides an upper limit that is strongly dependent on the halo size; ii) the Galactic wind lessens the $f$-volumes: either propagation is convection-dominated in this case, the origin depends only on the value of $L$ and $r_{\mathrm{w}}$, i.e. $V_{\mathrm{c}}$ and $K(E)$ - or it is escape-dominated and the geometrical upper limit (sole dependence on $L$, not $K$ ) is recovered; iii) spallations also systematically lessen the $f$-volumes: the heavier the nucleus, the larger its destruction rate, the closer it comes from. However, as a particle created in the halo is less likely to cross the disk, this effect is negligible compared to the wind for $r_{\mathrm{w}} \gtrsim r_{\mathrm{sp}}$. We show below that all these effects are more pronounced for annihilating SUSY than for evaporating PBH because the density profile $h_{\mathrm{DM}}(r, z)$ appears with a square.

\subsection{Dark matter distribution}

The dark matter distribution in our Galaxy is poorly known, and several dark matter profiles can be used. The first constraint is that the observed rotation curve of our Galaxy is almost flat beyond a few kpc from the center. For a spherical halo, it follows that the density decreases as $1 / r^{2}$ outside the central regions. In the inner regions, the situation is far from clear. Numerical simulations indicate that the central distribution of dark matter is cuspy, with a $r^{-\gamma}$ dependence with $\gamma \sim 0.5-1.5$ (Ghez et al. 1998), but this seems to be in contradiction with observations (Binney \& Evans 2001).
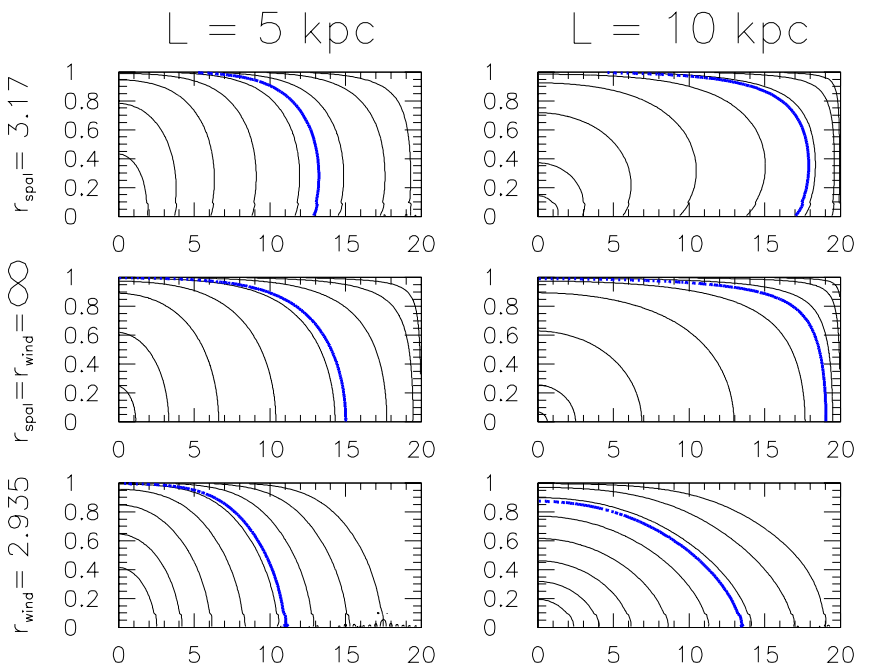

Fig. 2. Isodensity surfaces in the $(r, z)$ plane for $L=5 \mathrm{kpc}$ and $L=10 \mathrm{kpc}$ (side boundary $R=20 \mathrm{kpc}$ ). Inner contours correspond to $\mathrm{d} \mathcal{P}\left(r_{\mathrm{s}}, z_{\mathrm{s}} \mid O\right) / \mathrm{d}^{3} \boldsymbol{r}_{\mathrm{s}}=0.01 \mathrm{kpc}^{-3}$ and the contours are spaced by a factor $1 / 4$. From top to bottom, $r_{\mathrm{sp}}=3.17 \mathrm{kpc}$ (no wind), no wind and no spallations, $r_{\mathrm{w}}=2.935 \mathrm{kpc}$ (no spallations). These numbers correspond, respectively, for a reasonable choice of $K(E)$ at $1 \mathrm{GeV}$, to $\sigma_{\mathrm{sp}} \approx 100 \mathrm{mb}$ and $V_{\mathrm{c}} \approx 20 \mathrm{kpc} \mathrm{Myr}^{-1}$. The additional thick line in each panel delimitates contours $\mathcal{P}(\mathcal{V}(f) \mid O)=99 \%$.

In the absence of a clear answer to this problem, we use several profiles for the Dark Matter distribution, with the generic form

$h_{\mathrm{DM}}(r, z)=\left(\frac{R_{\odot}}{\sqrt{r^{2}+z^{2}}}\right)^{\gamma}\left(\frac{R_{\mathrm{c}}^{\alpha}+R_{\odot}^{\alpha}}{R_{\mathrm{c}}^{\alpha}+\left(\sqrt{r^{2}+z^{2}}\right)^{\alpha}}\right)^{\epsilon}$

where spherical symmetry has been assumed. Numerical simulations point toward singular profiles with $\gamma=1.5, \alpha=1.5$, $\epsilon=1$ and $R_{\mathrm{c}}=33.2 \mathrm{kpc}$ (Moore et al. 1999) or $\gamma=1, \alpha=1$, $\epsilon=2$ and $R_{\mathrm{c}}=27.7 \mathrm{kpc}$ (Navarro et al. 1996, hereafter NFW). We also considered an isothermal profile with $\gamma=0, \alpha=2$, $\epsilon=1$ and $R_{\mathrm{c}}=3 \mathrm{kpc}$ (the modified isothermal profile would give very similar results).

As already said, exotic SUSY particles (resp. PBH) are supposed to fill (resp. follow) the dark matter halo profile $h_{\mathrm{DM}}(r, z)$. However, the nature of the cosmic ray creation process is different in these two cases, leading to very different effective source terms, i.e. different weight $w(r, z)$ in Eq. (1). For evaporating Primordial Black Holes, the particle production is proportional to the density of the objects $w_{\mathrm{PBH}}(r, z) \propto h_{\mathrm{DM}}(r, z)$. In contrast, the production term for supersymmetric particles is proportional to the square of the density because two dark matter particles must be present for annihilation to occur. In this case $w_{\mathrm{SUSY}}(r, z) \propto h_{\mathrm{DM}}(r, z)^{2}$.

They are displayed in Fig. 3 both for SUSY and PBH weight (see above). The Moore and NFW profiles are singular at the Galactic center, so that the source term is much stronger there. The probability that a cosmic ray detected in the Solar neighborhood was emitted from this region is enhanced for these profiles. A crude estimate of this effect is obtained by a mere count of the effective (PBH or SUSY) source numbers in this critical region. For example, in the range [0-2] kpc, a Moore profile leads to an enhancement $\times 2.7$ 


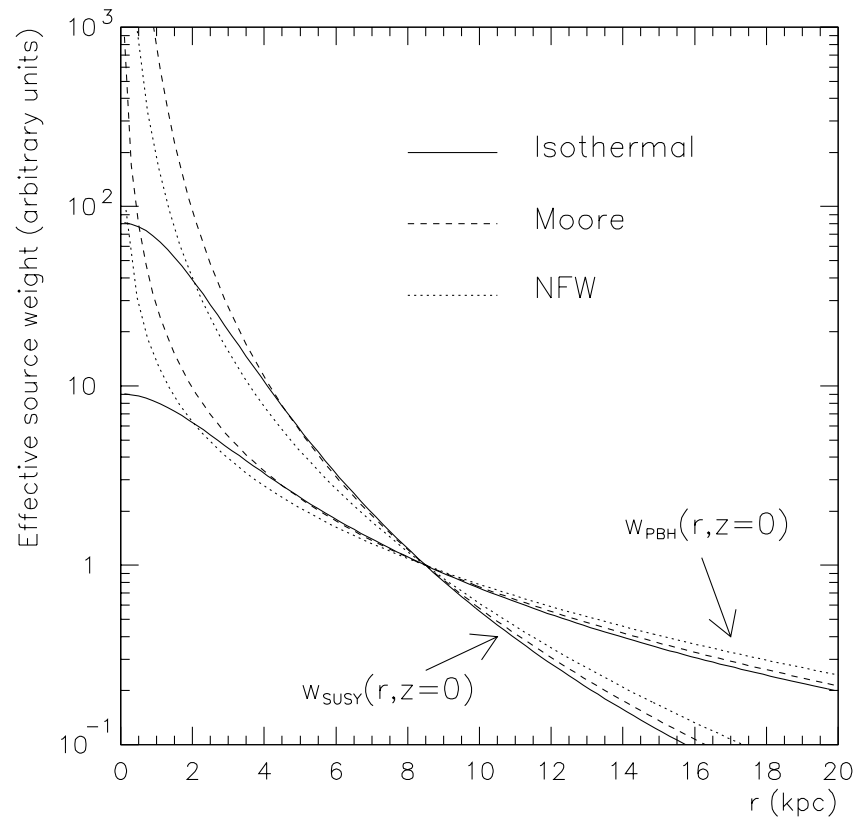

Fig. 3. Effective source weight (PBH or SUSY) for several profiles (see text).

for PBHs and $\times 90$ for SUSY annihilations, compared to the isothermal case. Stretching this interval decreases the enhancement factor, and for [0-4] kpc, it is respectively $\times 1.5$ and $\times 25$, and finally for [0-8] kpc, the numbers are $\times 1.1$ and $\times 20$. The enhancement is far smaller for PBH than for SUSY particles. Notice that the upper limits on the PBH density derived from antiproton flux measurements in Barrau et al. (2002) were of the same order of magnitude for an isothermal halo and for cuspy halos. This result is definitively not transposable to the SUSY case.

This is not the final word. The center of our Galaxy contains a supermassive black hole $(\mathrm{SBH})$ of a few $10^{6} M_{\odot}$. During its formation, it probably accreted the surrounding dark matter, leading to a local enhancement of the density. Gondolo \& Silk (1999, hereafter GS) found that if the SBH grows adiabatically in the center of the Galaxy, the cuspy profile $\left(\rho(r) \propto r^{-\gamma}\right.$ with $0<\gamma<2$ ) becomes spiky and $\rho(r) \propto r^{-A}$ with $2.25<A<2.5$ in a region of a few parsecs around the black hole. The presence of the spike would have dramatic consequences for several predictions of the signal from annihilating dark matter particles, e.g. $\gamma$ and neutrinos (Gondolo \& Silk 1999) or synchrotron emission of $e^{+} e^{-}$pairs (Gondolo 2000; Bertone et al. 2001). The signal coming from the direction of the Galactic Center is obtained by integrating along the line of sight, and the contribution of the central region is very different with or without a spike. In the case of the isothermal profile, the central region (around the SBH) contributes at the level of $\sim 10^{-9}$ whereas this contribution is greater than $\sim 10^{5}$ for a Moore profile (Gondolo $\&$ Silk 1999). However, these results are expected to be overoptimistic, and it is doubtful that such a spike exists in our Galaxy, as indicated by a more careful dynamical modelling of the SBH growth (Ullio et al. 2001). These authors review several effects (adiabatic growth versus instantaneous growth, models with off-centered black holes) and recover some results that were known before the Gondolo \& Silk paper: only the peculiar case in which the SBH forms adiabatically at the exact center of the dark matter profile can lead to an enhancement such as described in GS. Finally, in a recent study, Merritt et al. (2002) have observed that, taking into account the quite large probability that the Milky Way experienced a major merger in its history, the ensuing dark matter profile and resulting annihilation fluxes could be several order of magnitudes smaller than obtained with dark matter profile not disturbed by a SBH.

The points discussed above are mostly relevant for particles travelling in straight lines. For charged particles, due to the diffusive nature of propagation, the probability to come from a sphere $\mathcal{S}$ of radius $r=10 \mathrm{pc}$ around on the Galactic center $\left(\sim 8 \mathrm{kpc}\right.$ away) is $\int_{\mathcal{S}}\left(\mathrm{d} \mathcal{P} / \mathrm{d}^{3} \boldsymbol{r}_{\mathrm{s}}\right) \mathrm{d}^{3} \boldsymbol{r}_{\mathrm{s}}$, which is $\lesssim 10^{-10}\left(\mathrm{~d} \mathcal{P} / \mathrm{d}^{3} \boldsymbol{r}_{\mathrm{s}}\right.$ is given for example in Fig. 2). Due to the very narrow scale where the SBH may affect the distribution, even enhancement such as obtained in Gondolo \& Silk (1999) - and which is not very realistic - cannot yield a significant contribution for charged particles. Eventually, the dark matter profile remains of importance (isothermal or cuspy). In the following, most results will be presented for the isothermal case, the influence of the cusp being discussed at the end.

\section{3. $f$-volumes for SUSY and PBH weights and different values of $L$}

We now have all the elements to compute the $f$-volumes, inserting the source distributions described above in Eq. (1). The function entering the integral does not possess cylindrical symmetry, so that the full three-dimensional integral must be computed. We first neglect spallations and galactic wind to consider only the effect of $L$. This parameter is expected to play an important role, as the charged particles created outside of the magnetic halo of our Galaxy do not penetrate inside it and are not detected (Barrau et al. 2002, 2003). Figure 4 shows the $99 \%$-volumes in the Galactic plane $z_{\mathrm{s}}=0$ (upper panel), for the PBH and SUSY case. Their shape reflects the fact that the probability density has a maximum at $r_{\mathrm{s}}=R_{\odot}$, while the effective source distribution peaks at the Galactic center $O$. Because of the quadratic dependence on $h_{\mathrm{DM}}(r, z), \mathcal{V}_{\mathrm{SUSY}}(99 \%)$ is smaller than $\mathcal{V}_{\mathrm{PBH}}(99 \%)$. Three halo sizes are displayed $(L=2,5$ and $10 \mathrm{kpc})$ : for larger halos, the surfaces are more deformed towards the Galactic center (the contribution of this region to the flux is larger), whereas they remain grossly unaffected in the anti-center direction. This effect is less pronounced in the case of a PBH-like source distribution. The same contours are also plotted for $y_{\mathrm{s}}=0$ in the lower panel. The shapes are almost maximally distorted towards rectangular contours. This is less and less pronounced, as either $L$ is enhanced, or larger powers of $h_{\mathrm{DM}}(r, z)$ are chosen.

The figures above show clearly that we are only sensitive to a well-defined region of the source distribution: first to the region which is embedded in the diffusive halo, and then, even within this region, to a sub-region between the Galactic 


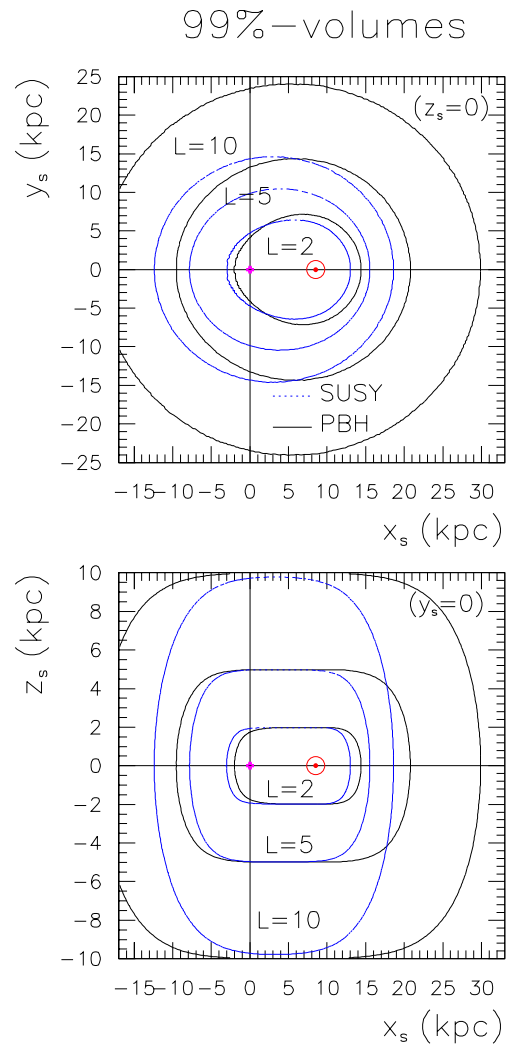

Fig. 4. Contours $\mathcal{P}\left(\mathcal{V}_{\mathrm{SUSY}} \mathrm{PBH} \mid R_{\odot}\right)=99 \%$ origin for $L=2, L=5$ and $L=10 \mathrm{kpc}$. $f$-volumes have been respectively evaluated with weight $w_{\mathrm{PBH}}(r, z) \propto h_{\mathrm{DM}}(r, z)$ (solid lines) and $w_{\mathrm{SUSY}}(r, z) \propto h_{\mathrm{DM}}^{2}(r, z)$ (dashed lines) for $R \gg L$, but the results remain mostly unchanged using $R=20 \mathrm{kpc}$ (but in case of a halo size $L=10 \mathrm{kpc}$ that requires $R=30 \mathrm{kpc})$. Upper panel: $V_{\mathrm{SUSY}}(99 \%)$ and $V_{\mathrm{PBH}}(99 \%)$ in the $x_{\mathrm{s}}-y_{\mathrm{s}}$ plane $\left(z_{\mathrm{s}}=0\right)$. Lower panel: same quantities but in the $x_{\mathrm{s}}-z_{\mathrm{s}}$ plane $\left(y_{\mathrm{s}}=0\right)$. In both panels, the dot marks the Galactic center, and $\odot$ denotes the Sun location (it is set to $R_{\odot}=8 \mathrm{kpc}$ ).

center and the Solar neighborhood. These sub-regions represent a fraction of the total number of sources given by

$f^{\mathrm{tot}}(L)=\frac{\int_{\mathcal{V}(99 \%)} w(r, z) \mathrm{d}^{3} \boldsymbol{r}}{\int w(r, z) \mathrm{d}^{3} \boldsymbol{r}}$,

where $\int w(r, z) \mathrm{d}^{3} \boldsymbol{r}$ is the total number of sources. It is also of interest to compare the number of sources located in the same sub-regions to the number of sources in the diffusive halo

$f^{\mathrm{cyl}}(L)=\frac{\int_{\mathcal{V}(99 \%)} w(r, z) \mathrm{d}^{3} \boldsymbol{r}}{\int_{\mathcal{V}_{\mathrm{cyl}}} w(r, z) \mathrm{d}^{3} \boldsymbol{r}} ;$

where $\mathcal{V}_{\text {cyl }}$ is the volume of the diffusive halo. The corresponding numbers are given in Table 1 for various halo sizes. The fraction $f^{\text {cyl }}(L)$ decreases with $L$, much faster for $\mathrm{PBH}$ than for SUSY. This can be understood as the number of contributors outside of the dark halo core radius rapidly vanishes for SUSY particles (see Fig. 1). As regards the results for $f^{\text {tot }}(L)$, we remark that this number is particularly small for $\mathrm{PBH}$, i.e. only a very small fraction of primordial halos distributed in the Galaxy contribute to the charged primary cosmic rays detected in the Solar neighborhood.
Table 1. Fraction of the number of exotic primaries emitted in $V_{\mathrm{PBH}}(99 \%)$ and $V_{\mathrm{SUSY}}(99 \%)$ for various $L$, compared to the total number of exotic primaries emitted either in the bounded geometry (halo size $L$ and radial extension $R=20 \mathrm{kpc}$ ) - denoted $f^{\text {cyl }}(L)-$, or in the whole dark halo - denoted $f^{\text {tot }}(L)$.

\begin{tabular}{lcccc}
\hline \hline & \multicolumn{2}{c}{$f^{\text {cyl }}(L)$} & \multicolumn{2}{c}{$f^{\text {tot }}(L)$} \\
& PBH / SUSY & \multicolumn{2}{c}{ PBH / SUSY } \\
\hline$L=10 \mathrm{kpc}$ & $\sim 1$. & $\sim 1$. & 0.023 & 0.76 \\
$L=5 \mathrm{kpc}$ & 0.70 & 0.85 & 0.010 & 0.54 \\
$L=2 \mathrm{kpc}$ & 0.31 & 0.60 & 0.002 & 0.21 \\
\hline
\end{tabular}

Table 2. Fraction of primaries $f^{\text {esc }}$ emitted from the (50-90-99)\%volumes of the cylindrical geometry (see above) that escape through upper and lower boundary located at $L=10 \mathrm{kpc}$ or $L=2 \mathrm{kpc}$ (for PBH and SUSY effective source distribution), before they can reach the Solar neighborhood.

\begin{tabular}{ccc}
\hline \hline & $L=10 \mathrm{kpc}$ & $L=2 \mathrm{kpc}$ \\
\hline$f_{\mathrm{PBH}}^{\text {esc }}: \mathcal{V}(50-90-99 \%)$ & $40-55-64 \%$ & $45-75-88 \%$ \\
$f_{\mathrm{SUSY}}^{\text {esc }}: \mathcal{V}(50-90-99 \%)$ & $49-52-55 \%$ & $59-92-95 \%$ \\
\hline
\end{tabular}

Finally, it is also interesting to give the fraction of primaries that escape before reaching the Solar neighborhood. It is defined as

$f^{\text {esc }}(\mathcal{V}) \equiv 1-f^{\operatorname{detect}}(\mathcal{V})=1-\frac{\int_{\mathcal{V}} w(r, z) N_{\mathrm{cyl}}\left(\boldsymbol{r} \mid R_{\odot}\right) \mathrm{d}^{3} \boldsymbol{r}}{\int_{\mathcal{V}} w(r, z) N_{\infty}\left(\boldsymbol{r} \mid R_{\odot}\right) \mathrm{d}^{3} \boldsymbol{r}}$,

where $N_{\text {cyl }}\left(\boldsymbol{r} \mid R_{\odot}\right)$ and $N_{\infty}\left(\boldsymbol{r} \mid R_{\odot}\right)$ are respectively related to the flux of particles detected at $R_{\odot}$, in the cylindrical geometry and in an unbounded space, from the same sources emitting from inside the volume $\mathcal{V}$. Estimations for $L=10 \mathrm{kpc}$ and $L=2 \mathrm{kpc}$ are compiled in Table 2. The trends are conform to intuition. Forming greater fractions of the detected flux requires more distant sources, the latter more easily escape through boundaries. For large diffusive halo $L$, the fraction that escape increases more quickly for PBH sources than for SUSY sources, whereas the converse is true for small halos. This is related to the fact that one has to compare the shape and typical extension of the source distribution to the parameter $L$. The fraction of primaries which are emitted inside $\mathcal{V}$ but which never reach the solar neighborhood is actually greater than $f^{\text {esc }}$, as even in the case of diffusion in unbounded space, there are many trajectories which start in $\mathcal{V}$ and never reach the Solar neighborhood (diffusion in three dimensions is a transient process).

\section{Realistic propagation parameters}

The previous section considered simplified diffusion situations with a typical value $K \sim 0.03 \mathrm{kpc}^{2} \mathrm{Myr}^{-1}$. Actually, $K(E)$ is energy dependent, and more precisely,

$K(E)=K_{0} \beta \mathcal{R}^{\delta}$.

Here, $\delta$ is the diffusion slope and $K_{0}$ the normalization of the diffusion coefficient. In a previous study (see Papers Ia, Ib), we show that various combinations of parameters $K_{0}, \delta$, diffusive halo height $L$ and Galactic wind magnitude $V_{\mathrm{c}}$ are equivalent, 
in the sense that they give a $\mathrm{B} / \mathrm{C}$ spectrum that is consistent with the observations. In this section, we use these combinations to provide a realistic range of values for $r_{\mathrm{w}}$ and $r_{\mathrm{sp}}$ and to explore the consequences on the origin of exotic primary antiprotons and antideuterons. The heavier antinuclei will not be considered here, as it was shown by Chardonnet et al. (1997) that their formation is suppressed because of the low probability of coalescence of many antinucleons.

\subsection{Parameter range allowed}

To compute the parameters introduced in Eqs. (5), the spallation cross sections of antiprotons and antideuterons are taken from the Particle Data Group ${ }^{1}$. In this work, we only consider spallation on pure hydrogen. It would be straightforward to take into account the spallations on the Helium component of the interstellar medium, but the effect is too small to be worth the complication. The four parameters $K_{0}, \delta, L$ and $V_{\mathrm{c}}$ are taken from our comprehensive study of standard secondary to primary B/C ratio (Maurin et al. 2002). Three values (two extremes and a medium value) have been retained for both the diffusion slopes ( $\delta=0.35,0.60$ and 0.85$)$ and the halo sizes ( $L=2 \mathrm{kpc}, L=6 \mathrm{kpc}$ and $L=10 \mathrm{kpc}$ ). We emphasize that the values of all these parameters come from the study of standard sources of cosmic rays and do not depend on the exotic sources, which do not produce $\mathrm{B}$ nor $\mathrm{C}$. We do not take reacceleration and energy losses into account in this work. These effects, though necessary to study the spectra of cosmic rays, are not so crucial here as they only amount to a redistribution of the cosmic rays at different energies. A particle detected at an energy of $1 \mathrm{GeV} /$ nuc was just created at a slightly different energy and its origin is not drastically different.

The values of $r_{\mathrm{sp}}$ and $r_{\mathrm{w}}$ are plotted in Fig. 5 for antiprotons and antideuterons. The left panel shows that propagation is convection-dominated $\left(r_{\mathrm{w}} \ll 1\right)$ at low energy when large $\delta$ values are considered and escape-dominated at all energies for small $\delta$. Notice that although at a given $\delta$, the quantity $r_{\mathrm{w}} / L$ is fairly independent of $L$, the origin is definitely not the same for $L=2 \mathrm{kpc}$ as for $L=10 \mathrm{kpc}$. The right panel shows that spallation is not the dominant effect for the light nuclei considered here. Only for large diffusion slopes $\delta$ and more particularly for antideuterons this effect becomes sizeable and comparable to the diffusive escape. The comparison of the two panels shows that spallations are always less efficient than convective wind or boundary escape. Finally, whatever the value of $\delta$, propagation is escape-dominated above a few tens of $\mathrm{GeV} /$ nuc and the origin of primary cosmic rays is solely dependent on the halo size.

\subsection{Antiprotons and antideuterons}

We are now able to draw the $f$-volumes for the realistic propagation parameters being considered. We focus on the antideuteron signal as it seems to be the most promising species to look for in cosmic rays. An interstellar energy of $1 \mathrm{GeV} /$ nuc is chosen; the nuclei that reach the detector are solar modulated so

\footnotetext{
1 http://pdg.lbl.gov/
}

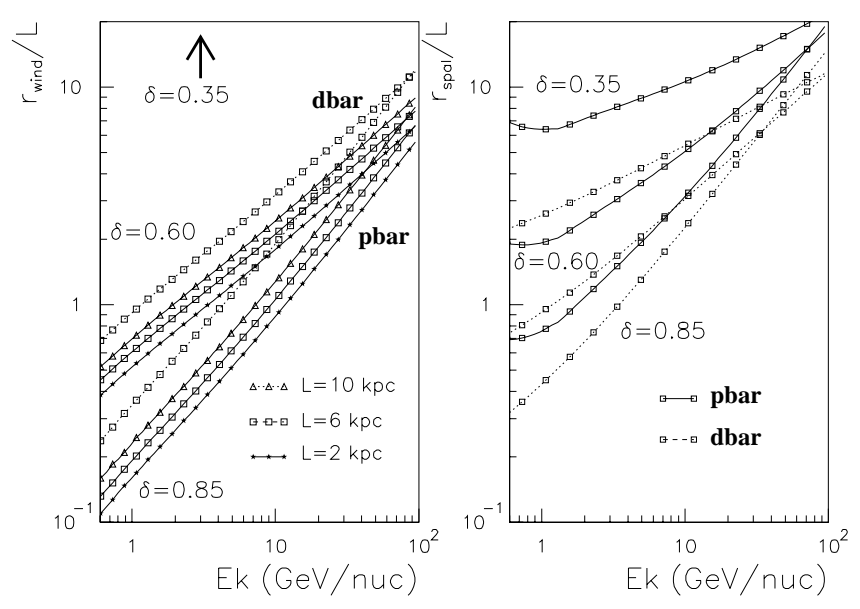

Fig. 5. Left panel: evolution of $r_{\mathrm{w}}$ as a function of kinetic energy per nucleus for primary $\bar{p}$ and $\bar{d}$; from top to bottom, $\delta=0.35, \delta=0.60$ and $\delta=0.85$. The parameter $r_{\mathrm{w}} / L \equiv \chi_{\mathrm{w}}$, as well as $r_{\mathrm{sp}} / L \equiv \chi_{\mathrm{sp}}$, are not very sensitive to the halo size $L$ (for $\bar{d}$, only $L=6 \mathrm{kpc}$ is displayed) but $r_{\mathrm{w}}$ and $r_{\mathrm{sp}}$ do. Right panel: $r_{\mathrm{sp}} / L$ as a function of $E_{k} /$ nuc for the same $\delta$ values and for the halo size $L=6 \mathrm{kpc}$. The values of $r_{\mathrm{w}}$ are different between $\bar{p}$ and $\bar{d}$ because they depend on rigidity (through $K$ ), i.e. on $Z / A$ (it is 1 for $\bar{p}$ and $1 / 2$ for $\bar{d}$ ). For $r_{\mathrm{sp}}$, there is an additional strong dependence on the species because of the destruction cross sections.

Table 3. $r_{\mathrm{w}}$ and $r_{\mathrm{sp}}$ for three halo sizes $L$ and three diffusion slopes $\delta$ : these numbers are for $1 \mathrm{GeV} / \mathrm{nuc}$ (interstellar energy) antideuterons.

\begin{tabular}{ccccc}
\hline \hline & $(\mathrm{kpc})$ & $\delta=0.35$ & $\delta=0.60$ & $\delta=0.85$ \\
\hline$L=10 \mathrm{kpc}$ & $r_{\mathrm{w}}=$ & $\infty$ & 8. & 2.9 \\
& $r_{\mathrm{sp}}=$ & 21. & 7.6 & 3.5 \\
$L=6 \mathrm{kpc}$ & $r_{\mathrm{w}}=$ & $\infty$ & 5.5 & 2.1 \\
& $r_{\mathrm{sp}}=$ & 15.5 & 5.5 & 2.6 \\
$L=2 \mathrm{kpc}$ & $r_{\mathrm{w}}=$ & $\infty$ & 2.1 & 0.85 \\
& $r_{\mathrm{sp}}=$ & 6. & 2.2 & 1.05 \\
\hline
\end{tabular}

that they are detected with a final energy of 400-800 MeV/nuc, where the signal is the more interesting. Table 3 summarizes the values of $r_{\mathrm{w}}$ and $r_{\mathrm{sp}}$ at this energy for antideuterons. The situation is very different for small or large $\delta$. For small values (corresponding roughly to a Kolmogorov power spectrum $\delta=$ $1 / 3)$, only spallations affect the propagation $\left(V_{\mathrm{c}}=0, r_{\mathrm{w}}=\infty\right)$ and this effect was shown to be weak; for large $\delta-$ the value $\delta=0.85$ is the one preferred in our $\mathrm{B} / \mathrm{C}$ analysis (Paper $\mathrm{Ib}$ ) models are convection-dominated though $r_{\mathrm{sp}}$ and $r_{\mathrm{w}}$ have about the same strength.

Figure 6 displays $\mathcal{P}_{\text {cyl }}(\mathcal{V}(f) \mid O)=99 \%$ for the values reported in Table 3. For $\delta=0.35$ (external contours), the geometrical (upper limit) contours are recovered. However, for larger $\delta$ (internal contours), these contours shrink. All comments made in Fig. 4 as regards halo size, or SUSY and PBH behavior, remain valid. Actually, the diffusion coefficient slope $\delta$, as $L$ for the geometrical limit, is a key parameter to trace back the CR origin, because of the values of $r_{\mathrm{w}}$ it implies, through $V_{\mathrm{c}}$ and $K_{0}$.

It is also of interest to have a closer look at the first $\%$ that contribute to the flux. As the $f$-volumes with $f \lesssim 50 \%$ correspond to isodensity contours that are quite insensitive to the 
99\%-volumes: dbar ( $1 \mathrm{GeV} /$ nuc)
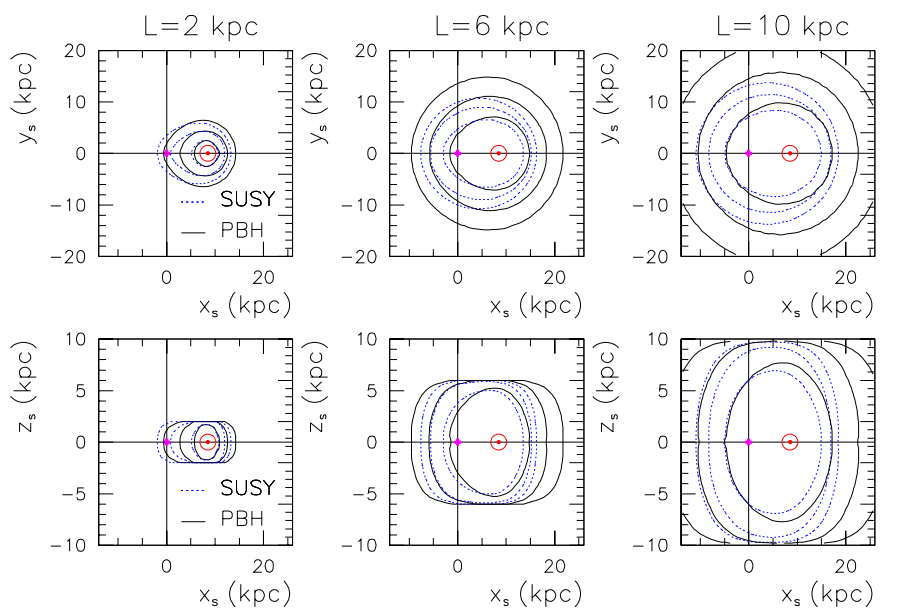

Fig. 6. 99\%-volumes for exotic primaries (no side boundaries). Upper panels: cut in the $z_{\mathrm{s}}=0 \mathrm{kpc}$ plane; lower panels: cut in the $y_{\mathrm{s}}=0 \mathrm{kpc}$ plane. Left panels correspond to $L=2 \mathrm{kpc}$, middle panels to $L=6 \mathrm{kpc}$ and right panels to $L=10 \mathrm{kpc}$. In each panel, we plot either the PBH case (solid lines) or the SUSY case (dotted lines). From external lines to internal lines correspond the values of the diffusion coefficient slope $\delta=0.35, \delta=0.60, \delta=0.85$.
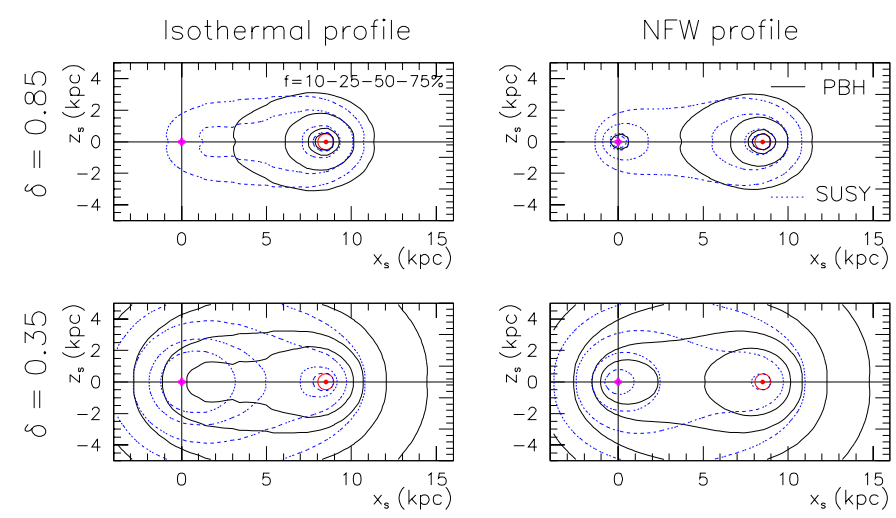

Fig. 7. $\mathcal{P}_{\text {cyl }}(\mathcal{V}(f) \mid O)=10 \%-25 \%-50 \%-75 \%$ for $L=10 \mathrm{kpc}$ in the $z_{\mathrm{s}}=0$ plane (except for the $75 \%$-volumes, other $f$-volumes with $f \lesssim 50 \%$ are not deformed by the boundaries so that they present symmetry around the $x_{\mathrm{s}}$ axis). Upper panels correspond to $\delta=0.85$, and lower panels to $\delta=0.35$. Both the PBH case (solid lines) and the SUSY case (dotted lines) are plotted.

boundaries (or to other effects) they present the axial symmetry around the $x_{\mathrm{s}}$ axis, so that a single cut through, e.g. the $x_{\mathrm{s}}-z_{\mathrm{s}}$ plane, delivers all the information about their shape. Figure 7 displays the $f$-volumes $f=10-25-50-75 \%$ for $L=10 \mathrm{kpc}$. The difference observed in Fig. 7 between small (lower panels) and large $\delta$ (upper panels) is readily explained: a large value of $\delta$ also corresponds to a large value of $K_{0}$ (see Maurin et al. 2003 for details), so that a greater wind is needed in order to prevent from too many spallations occurring at low energy. The net result is that the wind blows the particles away, reducing the effective zone from where they come. This is not the case for small $\delta$ where the geometrical limit (pure diffusion) is almost reached. The consequences for indirect dark matter searches are important. In the case of an isothermal profile (left panels), the particles created in the Galactic center have a small probability to reach the detector for large $\delta$, whereas the converse is true for small $\delta$. In the latter case, the predictions and the limits that can be put on a supersymmetric signal depend heavily on the central shape of the dark matter halo, which is precisely the part we know the least about. These contours for smaller diffusive halo sizes $L$ have not been presented; they have a smaller extent, meaning that we are less sensitive to the distribution of dark matter far from the Solar neighborhood. As a result, the question of the dark matter density profile cusp is less crucial for small $L$.

Similar contours for the NWF profile are drawn in the right panels of Fig. 7. Combining information from the above surfaces to the relative enhancement of sources going from the isothermal case to the cuspy case allows several complementary remarks: for small $\delta$, about half the SUSY Cosmic rays come from the range [0-3] kpc. Thus, the $\sim 50$ enhancement factor on the production provided by the cusp translates directly into a factor $\times 50$ in the detected flux. For PBH case, the origin is less localized and the enhancement factor is smaller, so that the net gain is more probably about $10-20 \%$. For large $\delta$, contours look like boxes encompassing both the Solar position and the Galactic center. In the SUSY case, the addition of a cusp strongly deforms the box towards the Galactic center, but it is not straightforward to estimate the enhancement without considering specific values for the diffusion parameters. For PBH, the contours, and hence the flux, are not expected to be very sensitive to the parameters. This discussion is of less importance for small halo sizes.

From the above discussion, it appears that the most important parameters are $L$ and $V_{\mathrm{c}} / 2 K$. The value $\delta=1 / 3$ (Kolmogorov spectrum) corresponding to $V_{\mathrm{c}}=0$, has been preferred these last years (see e.g. Strong \& Moskalenko 1998). However, our previous studies (Maurin et al. 2001, 2002,2003 ) show that large values of $\delta$, and non-null values of $V_{c}$, are preferred. This trend is confirmed the most recent results of Moskalenko et al. (2002) who now tend to prefer $\delta=0.42-0.52$. To conclude, if the value of $\delta$ happens to be large or more precisely if a strong Galactic wind is preferred, the discussions about the dark matter profile, including about the existence of a spike, are not so crucial. If conversely $\delta$ is small (no wind), the dark matter cusp as well as the exact location of the Solar system should be accurately known before exploring the SUSY parameter space.

Finally, all the remarks made for antideuterons in the previous sections apply as well for antiprotons. According to Fig. 5, for a given $\delta$ at a given energy, the corresponding $r_{\mathrm{w}}$ is about twice its antideuteron value. The resulting $f$-volumes are larger than those for antideuterons, but the conclusions remain the same.

\subsection{Electrons and positrons}

Exotic sources in the halo also emit electrons and positrons. Positrons are more promising to study supersymmetric signals as the background of standard positrons is much lower than electron's $\left(e^{+} /\left(e^{+}+e^{-}\right)<0.1\right)$, being predominantly secondary. Recently, the HEAT experiment (Coutu et al. 1999) reported 
Table 4. The quantity $r_{\text {loss }}(\mathrm{kpc})$ is given for three halo sizes $L$ and three diffusion slopes $\delta$ at the total energy $E=7 \mathrm{GeV}$.

\begin{tabular}{cccc}
\hline \hline & $\delta=0.35$ & $\delta=0.60$ & $\delta=0.85$ \\
\hline$L=10 \mathrm{kpc}$ & 1. & .65 & .48 \\
$L=6 \mathrm{kpc}$ & .85 & .55 & .41 \\
$L=2 \mathrm{kpc}$ & .53 & .35 & .26 \\
\hline
\end{tabular}

an excess at about $7 \mathrm{GeV}$ (see also the MASS-91 experiment, Grimani et al. 2002).

These particles are lighter than nuclei, so that they are subject to much stronger energy losses, due to synchrotron radiation and inverse Compton. This results in an effective lifetime given by (Aharonian et al. 1995; Atoyan et al. 1995; Baltz \& Edsjö 1999)

$\tau_{\text {loss }} \sim 300 \mathrm{Myr} \times \frac{1 \mathrm{GeV}}{E}$.

Aharonian et al. (1995) and Atoyan et al. (1995) showed that in that case, all boundaries have negligible effects on positrons and electrons above a few $\mathrm{GeV}$, and that the characteristic distance travelled by these species is $r_{\text {loss }} \sim \sqrt{K \tau_{\text {loss }}}$ (random walk through the tangled magnetic fields), or

$r_{\text {loss }} \sim 1 \mathrm{kpc} \times \sqrt{\frac{1 \mathrm{GeV}}{E}} \sqrt{\frac{K}{0.03 \mathrm{kpc}^{2} \mathrm{Myr}^{-1}}}$.

The result is an exponential cutoff that depends on the energy, i.e. the probability density reads $\mathrm{d} \mathcal{P}_{\text {rad }} / \mathrm{d}^{3} \boldsymbol{r}_{\mathrm{s}} \propto \exp \left(-r_{\mathrm{s}} / r_{\text {loss }}\right) / r_{\mathrm{s}}$ (see also Sect. 4.3 in Taillet \& Maurin 2003). In the case considered here of sources in the whole diffusive volume, the normalized probability density is given by

$\mathrm{d} \mathcal{P}_{\text {rad }}=\frac{\exp \left(-r_{\mathrm{s}} / r_{\text {loss }}\right)}{4 \pi r_{\mathrm{s}} \cdot r_{\text {loss }}^{2}} \mathrm{~d}^{3} \boldsymbol{r}_{\mathrm{s}}$

It is quite different from the case of a source distribution located in the disk only (see Eq. (12) in Taillet \& Maurin 2003). The resulting $f$-volumes (spheres) are given by

$\mathcal{P}_{\text {rad }}\left(r<r_{\text {lim }} \mid O\right)=1-\left(1+\frac{r_{\text {lim }}}{r_{\text {loss }}}\right) \exp \left(-\frac{r_{\text {lim }}}{r_{\text {loss }}}\right)$.

It means that sources that contribute to the fraction $f=$ (50-90-99)\% of the detected positrons emitted in the halo are located inside the sphere of radius $r_{\lim } \approx(1.7-4.8-6.6) \times$ $r_{\text {loss }}$. For the realistic values of $K(E)$ used above (see also Paper Ib), we compile in Table 4 the range covered by $r_{\text {loss }}$ at $E=7 \mathrm{GeV}$. Because of the very small scale involved along with the exponential decrease, $f$-volumes for positrons are expected to be only slightly deformed by the dark matter distribution, except for small $\delta$ and large $L$ whose $99 \%$-volumes extend up to $\sim 7 \mathrm{kpc}$.

It is possible now to make a few quantitative comments on the HEAT results and on the conclusion of Baltz et al. (2002) about this signal. They argued that, defining a boost factor related to the clumpiness of dark matter, one can accommodate with $e^{+}$data without enhancing too much the antiproton signal. The point is that antiprotons come from further than positrons, so that if a clump exists close to us, its contribution of antiprotons is averaged over a larger zone than positrons. A comparison of Figs. 6 and numbers presented above gives a relative distance

$r_{e^{+}, e^{-}}^{\text {origin }} / r_{\bar{p}, \bar{d}}^{\text {origin }} \sim 0.1$

for all reasonable stationary propagation models. However, considering large or small $\delta$, the effect of the clumpiness factor is expected to be different in different propagation models. Hence, the enhancement factor for the antiproton signal used in Baltz et al. (2002) should also depend on the diffusion efficiency, i.e. combination of diffusion plus convection (that is not considered in the above reference). To summarize, the relation between SUSY positron and antiproton signals is not straightforward, if the dark matter halo is clumpy. Thus it seems a hard task to combine constraints from these two different signals, unless they are obtained with the same analysis. Depending on $\delta$ and $L$, their origin is more or less local, and the size of the clumps as well as the typical distance between the clumps may be of importance.

\section{Summary and conclusions}

This paper analyzes the spatial origin of exotic particles created from a dark matter profile. We presented the $f$-volumes inside which a given fraction of the cosmic rays detected in the Solar neighborhood were emitted. At high energy $(E \gg 1 \mathrm{GeV} /$ nuc), the shape of the isodensity surfaces is set by the geometry of the diffusive halo, in particular on its height $L$, the influence of the side boundary at $r=R$ being small. We then showed that the $f$-volumes defined are smaller when spallations and convection are taken into account, but in a very different way: for particles in the diffusive halo, the wind exponentially decreases the probability of reaching the Galactic plane, whereas spallations have about a null effect on the latter. The parameters $L$ and $2 V_{\mathrm{c}} / K$ indicate whether the propagation is convection or escape-dominated. In Table 5 we summarize the parameters that act as a cut-off in various situations. Two source distributions for the isothermal dark matter profile were considered: production related to the density of the source (e.g. PBH evaporation), or production related to the square of the density of the sources (e.g. SUSY annihilation). The 99\%-volumes are strongly stretched toward the Galactic center, corresponding to the maximum of the source distribution. This follows from the competition between the effective source which is maximum at the Galactic center, and the probability density which steadily decreases from our position $R_{\odot}$ to reach $\sim 10^{-4}-10^{-5} \mathrm{kpc}^{-3}$ for purely diffusive regime (or even less when convection is included) at the Galactic center. The fluxes in the Solar neighborhood are found to be far more sensitive to the dark matter profile in the SUSY case than in the PBH case. In both cases, the side boundary of the diffusive volume is observed to play a negligible role as long as $R \gtrsim 20-30 \mathrm{kpc}$.

As a last step, realistic propagation parameters were implemented, and the key parameters were found to be the halo size $L$ and the diffusion slope $\delta$ (actually $V_{\mathrm{c}} / K_{0}$ ). For the species considered here (antiprotons and antideuterons), spallations always play a negligible role in the origin. It was found that this 
Table 5. Summary of the typical cut-off in $z$ and $r$ directions beyond where a cosmic ray cannot originate. The sole parameters that determine these cut-offs are $L$ (halo size) and/or $\chi_{\mathrm{w}} \equiv r_{\mathrm{w}} / L$ - related to the convective wind $V_{\mathrm{c}}$ via $r_{\mathrm{w}}=2 K / V_{\mathrm{c}}$, or $r_{\text {loss }}$ related to the effective life-time for positrons and electrons.

\begin{tabular}{cccc}
\hline \hline Cut-off & $\begin{array}{c}\text { Escape-dominated } \\
\left(\chi_{\mathrm{w}} \gg 1\right)\end{array}$ & $\begin{array}{c}\text { Convection-dominated } \\
\left(\chi_{\mathrm{w}} \ll 1\right)\end{array}$ & $\begin{array}{c}\text { Losses-dominated } \\
\left(e^{-} e^{+} \gtrsim \mathrm{GeV}\right)\end{array}$ \\
\hline Halo & $L$ & $L^{*} \approx 3 K / V=3 r_{\mathrm{w}} / 2$ & $\approx 5 r_{\text {loss }}$ \\
Radial & $\min (R, 3 L)$ & $\min \left(R, 3 L^{*}\right)$ & $\approx 5 r_{\text {loss }}$ \\
\hline
\end{tabular}

origin is far more local in case of large $\delta$ and small $L$ than in case of small $\delta$ and large $L$. Moreover, the shape of the dark matter distribution near the Galactic center does not matter so much for the PBH case, whereas it may be crucial for SUSY annihilating particles. We emphasized that in any discussion of the annihilation signal in charged particles, the propagation parameter $\delta$ or more precisely, the presence of a Galactic wind, should be considered, with the same importance of the parameter $L$ or the choice of the dark matter profile.

Two last points are worth noting. First, even though the work presented here does not allow a quantitative estimation of the effect of possible clumpiness of the dark matter halo (for SUSY annihilations), we observed that the comparison between the electron and antiproton SUSY signals should involve a careful inspection of the corresponding boost factors. Second, whereas the use of $\mathrm{B} / \mathrm{C}$-induced propagation parameters is justified for standard antiprotons (corresponding $f$-surfaces can be seen in Taillet \& Maurin 2003), there is no guarantee that these parameters are valid in the $f$-volumes depicted here.

Acknowledgements. This work has benefited from the support of PICS 1076, CNRS and of the PNC (Programme National de Cosmologie).

\section{Appendix A: Numerical evaluation of the point source solution in Bessel basis}

One needs to evaluate numerically point source solutions such as

$N_{\delta}^{\mathrm{cyl}}(r, z)=\frac{1}{\pi K R} \sum_{i=1}^{n_{\mathrm{tronc}}} \frac{J_{0}\left(\zeta_{i} r / R\right)}{\zeta_{i} J_{1}^{2}\left(\zeta_{i}\right)} \frac{\sinh \left[\zeta_{i}(L-z) / R\right]}{\cosh \left(\zeta_{i} L / R\right)}$.

In the above expression, $(r, z)$ is the position of the $\delta$ source in polar coordinate and $R$ is the radial extension of the Galaxy. $N_{i}^{\text {cyl }}(z)$ can be evaluated for each $i$ and need to be summed till the $n_{\text {tronc }}$ th order, which should formally tend to infinity. For evident reasons, $n_{\text {tronc }}$ is chosen to be the smallest possible with the constraint that the rebuilt series $N_{\delta}^{\mathrm{cyl}}(r, z)$ has reached a good convergence. In the case of $\delta(\boldsymbol{r})$ point source, profiles are singular near the source and convergence of the series appear to be very slow. The ansatz depicted in Taillet \& Maurin (2003) is useless as soon as sources are outside the disk. We present below two alternatives to evaluate this sum.

\section{A.1. Average value of the oscillating series with $r$}

In analogy with classical Fourier analysis, resummation of coefficients provide oscillating behavior around the true value.
This can be understood if we recall that at the $n$th order, the function added is $\propto J_{0}\left(x \equiv \zeta_{n} \rho\right)$ : $\rho$ lying in [0-1], the argument of $J_{0}$ takes values $x=\left\{\zeta_{1}, \zeta_{2}, \ldots \zeta_{n}\right\}$, i.e. at the $n$th order, the corrective function has $n$ roots. Thus convergence will be more quickly reached if for a given order $n_{\text {cutoff }}$, instead of evaluating $N_{\delta}^{\mathrm{cyl}}(r, z)$, one averages

$N_{\delta}^{\mathrm{cyl}}\left(r_{n}, z\right)=\frac{N_{\delta}^{\mathrm{cyl}}\left(r_{n-1}, z\right)+N_{\delta}^{\mathrm{cyl}}\left(r_{n+1}, z\right)}{2}$

where $r_{n-1}, r_{n}$ and $r_{n+1}$ are ordered realizations of $r$. The sole condition is that the $\left\{r_{n}\right\}^{n=1, \ldots n_{\text {cutoff }}}$ belong to the grid $r=$ $\left\{0, R /\left(2 n_{\text {cutoff }}\right), 2 R /\left(2 n_{\text {cutoff }}\right), \ldots, R\right\}$, i.e. $2 n_{\text {cutoff }}$ linear steps between 0 and 1 . To summarize, around the oscillating value, if the appropriate step is chosen, it ensures that the averaged two points are not both above or below the true value, and furthermore, that two opposite extrema of the oscillating function are averaged.

\section{A.2. Step-like source: $\theta$ function}

An alternative way is to consider solution from a step-like source, e.g. $\theta(a-r)$, in order to smooth the problematic behavior observed near the origin for the $\delta$ source. With the suitable normalization in the source term, i.e.

$q_{\theta}(r, z)=\frac{\theta(a-r)}{\pi a^{2}} \delta(z)$

and using the property $\int \rho J_{0}(\rho) \mathrm{d} \rho=\rho J_{1}(\rho)$, it leads to a solution which is equivalent to the delta solution $N_{\delta}^{\text {cyl }}(r, z)-$ Eq. (A.1) -, as long as the distance $r_{\mathrm{o}}$ of the observer $X_{\mathrm{o}}$ from the source satisfies the relation $r_{\mathrm{o}} \gg a$. The Bessel coefficients of $\delta$ and $\theta$ solutions are related through

$N_{i}^{\theta}\left(z_{\mathrm{o}}\right)=2 \times \frac{J_{1}\left(\zeta_{i} a / R\right)}{\left(\zeta_{i} a / R\right)} \times N_{i}^{\delta}\left(z_{\mathrm{o}}\right)$.

The acceleration of convergence can be understood as, in Eq. (A.2), the additional term behaves at least as $1 / i\left(J_{1}\right.$ is bounded and $\left.\zeta_{i} \approx i \pi\right)$. Here $a$ should be taken such as to verify $a / R \ll 1$ (with $R=20 \mathrm{kpc}$ for the Galaxy, one can safely take $a \sim 10 \mathrm{pc})$.

Thus, a $\theta$-like source slightly underestimates the result close to $\boldsymbol{r}=\boldsymbol{r}_{\mathrm{s}}$, but this zone corresponds to very small volumes that add a negligible contribution when one evaluates integrated probabilities. For practical purposes, both methods (average or $\theta$ source) give the desired results with about the same number of Bessel functions, i.e. $n_{\text {cutoff }} \sim 100$. 


\section{References}

Aharonian, F. A., Atoyan, A. M., \& Völk, H. J. 1995, A\&A, 294, L41 Atoyan, A. M., Aharonian, F. A., \& Völk, H. J. 1995, Phys. Rev. D, 52,3265

Baltz, E. A., \& Edsjö, J. 1999, Phys. Rev. D, 59, 23511

Baltz, E. A., Edsjö, J., Freese, K., \& Gondolo, P. 2002, Phys. Rev. D, 65,63511

Barrau, A., Boudoul, G., Donato, F., et al. 2003, A\&A, 398, 403

Barrau, A., Boudoul, G., Donato, F., et al. 2002, A\&A, 388, 676

Beach, A. S., Beatty, J. J., Bhattacharyya, A., et al. 2001, Phys. Rev. Lett., 87, A261101

Bergström, L., Edsjö, J., Gondolo, P., \& Ullio, P. 1999a, Phys. Rev. D, 59,43506

Bergström, L., Edsjö, J., \& Ullio, P. 1999b, ApJ, 526, 215

Bertone, G., Sigl, G., \& Silk, J. 2001, MNRAS, 326, 799

Binney, J. J., \& Evans, N. W. 2001, MNRAS, 327, L27

Boezio, M., Bonvicini, V., Schiavon, P., et al. 2001, ApJ, 561, 787

Bottino, A., Donato, F., Fornengo, N., \& Salati, P. 1998, Phys. Rev. D, 58, 123503

Bottino, A., Favero, C., Fornengo, N., \& Mignola, G. 1995, APh, 3, 77

Coutu, S., Barwick, S. W., Beatty, J. J., et al. 1999, APh, 11, 429

Donato, F., Fornengo, N., \& Salati, P. 2000, Phys. Rev. D, 62, 43003

Donato, F., Maurin, D., Salati, P., et al. 2001, ApJ, 563, 172

Ghez, A. M., Klein, B. L., Morris, M., \& Becklin, E. E. 1998, ApJ, 509,678
Gondolo, P. 2000, Phys. Lett. B, 494, 181

Gondolo, P., \& Silk, J. 1999, Phys. Rev. Lett., 83, 1719

Grimani, C., Stephens, S. A., Cafagna, F. S., et al. 2002, A\&A, 392, 287

Jungman, G., \& Kamionkowski, M. 1994, Phys. Rev. D, 49, 2316

Maeno, T., Orito, S., Matsunaga, H., et al. 2001, APh, 16, 121

Maki, K., Mitsui, T., \& Orito, S. 1996, Phys. Rev. Lett., 76, 3474

Maurin, D., Cassé, M., \& Vangioni-Flam, E. 2003, APh, 18, 471

Maurin, D., Donato, F., Taillet, R., \& Salati, P. 2001, ApJ, 555, 585 (Paper Ia)

Maurin, D., Taillet, R., \& Donato, F. 2002, A\&A, 394, 1039 (Paper Ib)

Merritt, D., Milosavljević, M., Verde, L., \& Jimenez, R. 2002, Phys. Rev. Lett., 88, 191301

Moore, B., Ghigna, S., Governato, F., et al. 1999, ApJ, 524, L19

Moskalenko, I. V., Strong, A. W., Ormes, J. F., \& Potgieter, M. S. 2002, ApJ, 565, 280

Navarro, J. F., Frenk, C. S., \& White, S. D. M. 1996, ApJ, 462, 563

Orito, S., Maeno, T., Matsunaga, H., et al. 2000, Phys. Rev. Lett., 84, 1078

Rudaz, S., \& Stecker, F. W. 1988, ApJ, 325, 16

Stecker, F. W., \& Tylka, A. J. 1989, ApJ, 336, L51

Strong, A. W., \& Moskalenko, I. V. 1998, ApJ, 509, 212

Taillet, R., \& Maurin, D. 2003, A\&A, 402, 971

Ullio, P., Zhao, H., \& Kamionkowski, M. 2001, Phys. Rev. D, 64, 43504

Wells, J. D., Moiseev, A., \& Ormes, J. F. 1999, ApJ, 518, 570 